\title{
Evaluation of Colour Versions of Wild Sage (Salvia nemorosa L.)
}

\author{
Kaprinyák, T. ${ }^{1}$, Koroknai, J. ${ }^{5}$, Zsiláné, A. A. ${ }^{2}$, Szakadát, Gy. ${ }^{3}$, Lévai, P.4, Kováts, Z.† and Fári, M. ${ }^{1,3}$ \\ ${ }^{1}$ Ornamental Crops Department, Debrecen Uni Böszörményi Ú. 138, 4032 Debrecen \\ ${ }^{2}$ Agricultural Technical and HighSchool, Debrecen Uni, Debrecen-Pallag \\ ${ }^{3}$ Plant Biotechnology Dept., Debrecen Uni., Böszörményi Ú. 138, 4032 Debrecen, Hungary \\ ${ }^{4}$ Ornamental Crops Department, College of Kecskemét \\ ${ }^{5}$ MOP Biotech Ltd., Nyíregyháza
}

${ }^{1}$ Corresponding author: Kaprinyák, T. (kaprinyak.tunde@ agr.unideb.hu)

\begin{abstract}
Summary: In the continental weather zone, more and more frequently occurring extreme conditions require continuous renewal of the market which generates constant challenge for the ornamental plant breeders. Most of the traditionally used decorative ornamental plants are sensitive to these extreme conditions. In 2001, Department of Plant Biotechnology, Debrecen University initiated an interdisciplinary breeding program in collaborations with Zoltan Kovats (he dealt with hungarian drought-tolerant plant species) to produce new or reintroduce forgotten drought-tolerant ornamental species into public parks and roadsides.

From $~ 900$ species of Salvia genus, Salvia nemorosa L. has been known as a medical plant, however, because of its high adaptation ability and decorative nature it is a highly recommended ornamental plant as well. Salvia nemorosa L. has a low maintenance, extremely droughttolerant, fast growing plant, generates proper cover, and highly competing weeds on roadsides.

Nowadays, 50-60 varieties are available; however this number could be increased by new hybrids. Great morphological and colour variation could be seen within the species, from different white to deep violet. The main goal of this research is the production of elite lines with wide colour and morphological variation in wild sage. We have already obtained 25 different clones for further investigation without eliminating the original plants generating an in vitro gene bank as it has been done by Italian breeders.
\end{abstract}

Keywords: climate change; ornamentals; Wild sage; colour versions; preservation

\section{Introduction}

Today, dynamic changes could be observed on the ornamentals plant market. The large usability, high-value ornamental species and varieties are preferred. Besides this, the global climate change also is a limiting factor for the ornamental plants cultivation. Most of applied ornamental plant species imported from other countries. Furthermore, adapting to different climates and adapting to the local conditions cause problems for these plants. The early autumn and late spring frosts and drought conditions are big challenges for the foreign ornamental plant species which are not able to provide features as promised by the catalogue. Meanwhile, the selected plants from native species have higher drought tolerance, disease and pest resistance could be adapted to the local conditions. Wild sage meets these requirements. Based on these facts, it could be noticed that, going back to native sources offer great possibility expanding the ornamental plant assortment (Kaprinyák et al., 2012).

Wild sage, as an ornamental plant can grow within a highly capacity of adaptation. Because of excellent drought- tolerant capability, it is suitable for extensive conditions. Roots can penetrate through the upper $30 \mathrm{~cm}$ layer of soil, therefore, it can utilize well the soil water supply. After planting, wild sage can provide sufficient coverage to inhibit the growth of weeds. Wild sage could be found in parks, along highways and in gardens, which is due to its excellent adaptability (Kaprinyák et al., 2012).

Over 900 species of the Salvia genus exist in the world. Some species are used for medicinal or horticultural production (Ruffoni et al, 2004). Originally, wild sage inherits from Mediterranean region. Wild sage can be found in Europe from agricultural areas to hills and mountains of the Pontic-Pannonian flora (Hegi, 1926).

From botanical point of view, wild sage has simple bracts and same size hairy sepals (Figure 1). On the long and spear-edged, leaves are not glandular hairs. The gray-green leaves have special sage smell (Király, 2009). Anthocyanin discoloration often can be seen on the erect branching stems at the beginning of the growth. The $8-14 \mathrm{~mm}$ long, usually purplish blue, sometimes white or pink upper lips have two pieces $5 \mathrm{~mm}$ long slightly drooped stamens (Cervelli, 1999). According to Soó (1968), the stem and the inflorescence axis 
of wild sage are downy gray. Wild sage is a member of the Lamiaceae family. Its height can be changed between 20-70 $\mathrm{cm}$. The colour versions of wild sage were emphasized by Soó (1968) as follows:

-1. albiflora white lips

-1. badacsonyensis rose lips

-1. elővölgyensensis lilac lips

-1. purpurea magenta lips

-1. coerulea pale blue lips

Lip flowers are blooming from May to autumn frosts. The nectar is producing continuously in the long blooming period, which is important food source for butterflies. From horticultural aspect, the most important considerations are the ornamental value, shape, size and the annual blooming duration and the frequency (Cervelli, 1999).

This valuable and unfairly undervalued plant is considered by botanists relicts. The current variety assortment consists of more than fifty varieties, but it continues to grow by hybridization. Three main color variations including white, pink and purple are mentioned in the botanical descriptions, but beside the basic colours, there are several different variations in the wild plants. Szaniszló Priszter was the first who published purple colorvariant of wild sage (Kováts, 2010). Zoltán Kováts breeded a large and a medium-sized, dark pink version from wild plant in Budatétény (Hungary). Natural vegetation mixture species can be also observed beside the basic species. $S$. $x$ betonicifolia Etl. (S. nemorosa $x$ nutans) and the $S . x$ sylvestris L. ( $S$. nemorosa $x$ pratensis) belonging to the mixture species (Soó, 1968).

The spontaneously appearing and disapperaring botanical shape and colour variations (lusus forms) of natural populations are still unknown, therefore our main goal is to map and evaluate them using by botanical and genetic methods.

\section{Materials and Methods}

In 2001, Department of Plant Biotechnology (Debrecen Uni) launched in collaboration with Zoltan Kovats an ornamental plant breeding program for drought-tolerant plant species, which involves the research of forgotten undervalued, but for outdoor use potentially suitable ornamental plants. This is achieved as an interdisciplinary activity (botany, genetics, breeding and biotechnology).

Based on the methods and results of our Italian colleagues (Cervelli and Ruffoni). the research closest to our activities standing, we can continue in our work. Examination of Cervelli and his colleagues (1999) included a germination tests, evaluation of new genotypes and varieties, of possibility of using cut and potted plants, case studies of micropropagation and plant pathology.

In 2010, our colleagues (Department of Plant Biotechnology, Debrecen Uni) with Zoltán Kováts visited some domestic natural wild sage populations, where some valuable shape and colour variations may live. The next year, we continued in adding and mapping of the area on the basis of the research of our internationally renowned breeder, Zoltan Kovats. This work have been achieved at the end of the flowering phenological phase (Fari, 2011).

The different shape and colour variations can live on the original location with the in vivo method. It could be collected the broke cuttings from wild population on the spot substance. The mother stems and clones can grow on without damaging the biodiversity on the new spot. We are evaluating the new versions continually.

The valuable horticultural varieties were planted (lusus forms) into the DE AGTC DTTI Demonstration Garden, which evaluated from point of morphological view in 2011. After evaluating the clones, the characteristics of possessing particular is selected specimens, which we would like to propagate with in vivo and in vitro methods in the future.

In the flowering period we recorded the special stamps continously and we counted the amount of flowers on the stems. Botanists have not observed yet the colour difference of leaves, inflorescence axis, the upper and the lower lips and the length of inflorescence axis. These properties may be important in term of usability. We determined the differences of the inflorescence axis of clones from size categories: short $0-10 \mathrm{~cm}$, medium 10-20 cm and above 20 cm long.

\section{Results}

From the ornamental point of view, the local flora hides valuable species. Zoltan Kovats started the wild sage colour range expansion. Our main goal is to continue this work. According to our knowledge, morphologically the wild sage has a large variability. The wild sage versions from the natural vegetation collected have been, as lusus forms (spontaneously appearing and disapperaring botanical shape and colour versions) the created clones have appeared next the three primary colours, new unique shape and colour versions. Evaluation of the collected, different wild sage shape and colour versions was done in 2011 (Kaprinyák et al., 2012). The Table 1 shows genetic variability of the different versions, which are still unknown for botanists and gardeners. According to the horticultural use, the most important stamps for us are the colour of leaf, inflorescence, axis and colour variation and the shape of the lips. These attributes were monitored in the summer of 2012 with priority attention (Table 2). Leaf colour of the examined stems and clones is usually pale green or green. The direction of the horticultural use was determined by the length of inflorescens axis. Colour of inflorescence axis has not been included in botanical descriptions yet, in turn this stamp has an important role in the association. The form of the lips is a new, interesting result, because the shape of lips of inflorescence axis is not mentioned in the descriptions. The upper and lower lips, furthermore the bracts and sepals colour can vary from below and above. The botanists know 


\begin{tabular}{|c|c|c|c|c|c|c|c|c|c|c|c|c|c|c|c|c|c|c|c|c|c|c|c|c|c|c|c|c|c|c|c|}
\hline $\begin{array}{l}0 \\
\vdots \\
z \\
\infty \\
\infty\end{array}$ & $\stackrel{\infty}{-}$ & 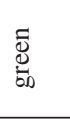 & & & $\begin{array}{l}\tilde{\Xi} \\
\overline{0} \\
\dot{5} \\
\dot{\nabla}\end{array}$ & 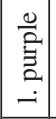 & & & 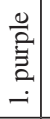 & & & 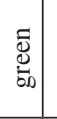 & 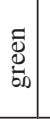 & $\frac{\stackrel{0}{a}}{\frac{1}{2}}$ & 产 & & & & & & & & & & & & & & & & \\
\hline $\begin{array}{l}n \\
0 \\
z \\
\infty\end{array}$ & 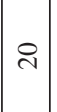 & 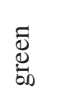 & & $\star$ & 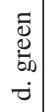 & & $x$ & & $\frac{0}{\frac{0}{3}}$ & & & 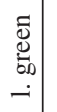 & 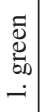 & 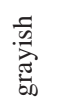 & 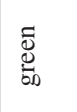 & $\begin{array}{l}\overline{\tilde{n}} \\
\mathbf{Z} \\
\tilde{S}\end{array}$ & $a$ & 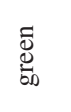 & & $x$ & 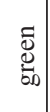 & $\mid \begin{array}{l}0 \\
\stackrel{2}{0} \\
- \\
-\end{array}$ & & & $\begin{array}{l}0 \\
\stackrel{0}{0} \\
-\end{array}$ & & & 竞 & 总 & $\mid$ & 足 \\
\hline $\begin{array}{l} \pm \\
z \\
z \\
\infty\end{array}$ & $\ddot{\lambda}$ & 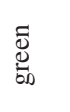 & $x$ & $\star$ & 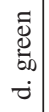 & 章 & & & $\frac{0}{3}$ & & & 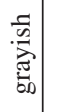 & \begin{tabular}{c|}
$\bar{\Xi}$ \\
$\stackrel{0}{0}$ \\
$\vdots$ \\
- \\
-
\end{tabular} & 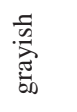 & 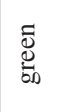 & 总 & $\stackrel{0}{\circ}$ & $\begin{array}{l}\bar{\Xi} \\
\text { एँ. }\end{array}$ & & $x$ & 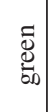 & 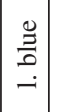 & & & \begin{tabular}{l}
0 \\
$ٍ$ \\
$ٍ$ \\
\hdashline
\end{tabular} & & $x$ & 党 & 咅 & 产 & 总 \\
\hline $\begin{array}{l}m \\
z \\
z \\
\infty\end{array}$ & $\because$ & 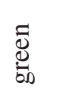 & & & 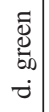 & 章 & & & $\stackrel{\frac{9}{3}}{3}$ & $\star x$ & & \begin{tabular}{c|c}
$\overline{\tilde{D}}$ \\
$\stackrel{\Delta}{\omega}$ \\
$\stackrel{-}{0}$
\end{tabular} & 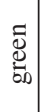 & 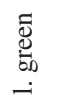 & $\begin{array}{l}\bar{\Xi} \\
\bar{\Xi} \\
\bar{b}\end{array}$ & 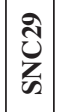 & 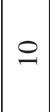 & $\frac{0}{2}$ & & 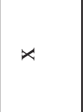 & 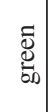 & $\mid \begin{array}{c}\frac{0}{2} \\
\frac{2}{2} \\
\stackrel{0}{2} \\
\stackrel{-}{2}\end{array}$ & & & $\begin{array}{l}\stackrel{0}{2} \\
\stackrel{\bar{z}}{2}\end{array}$ & & & 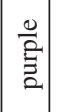 & $\begin{array}{l}\frac{0}{2} \\
\frac{2}{2} \\
\end{array}$ & 产 & 号 \\
\hline$\underline{z}$ & & & & & & & & & & & & & & & & 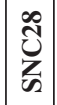 & $m$ & 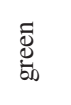 & & & 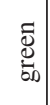 & $\mid \begin{array}{l}0 \\
\stackrel{2}{0} \\
\stackrel{2}{-}\end{array}$ & & $\times$ & $\begin{array}{l}\stackrel{0}{ٍ} \\
\stackrel{0}{\circ} \\
-\end{array}$ & & $x$ & 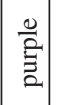 & 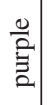 & 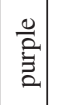 & 号 \\
\hline $\bar{z}$ & & & & & & & & & & & & & & & & $\begin{array}{l}\hat{N} \\
Z \\
Z \\
\infty\end{array}$ & \&ి & 言总 & & & 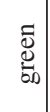 & 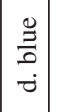 & & $\times$ & $\begin{array}{l}\varrho \\
ٍ \\
\dot{0} \\
\dot{0}\end{array}$ & & & 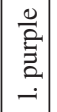 & $\begin{array}{l}\frac{0}{2} \\
\frac{2}{2} \\
\end{array}$ & 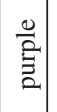 & 号 \\
\hline$\underset{m}{\stackrel{a}{z}}$ & & & & & & & & & & & & & & & & 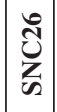 & 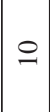 & 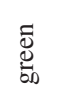 & & $x$ & $\begin{array}{l}\bar{\Xi} \\
\bar{\Xi} \\
\bar{b}\end{array}$ & 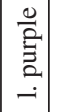 & & & 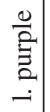 & $\times$ & & 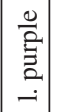 & $\begin{array}{l}\frac{0}{a} \\
\stackrel{a}{2} \\
\end{array}$ & 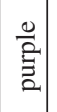 & 品 \\
\hline 妾 & & & & & & & & & & & & & & & & 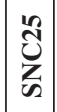 & ¿ & 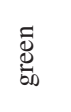 & & & $\begin{array}{l}\overline{\bar{v}} \\
\bar{\Delta} \\
\bar{b}\end{array} \mid$ & $\mid \begin{array}{c}0 \\
\frac{2}{2}\end{array}$ & & $\times$ & $\frac{0}{\frac{\partial}{\partial}}$ & & $\times$ & 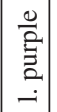 & 品 & 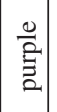 & 党 \\
\hline $\begin{array}{l}\infty \\
Z_{\infty}\end{array}$ & & & & & & & & & & & & & & & & 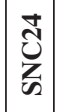 & त) & $\begin{array}{l}\text { एँ๊ } \\
\stackrel{\Delta}{0}\end{array}$ & $\times$ & $x$ & 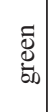 & 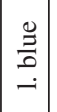 & & & $\begin{array}{l}\stackrel{0}{ٍ ٍ ٍ ٍ ~} \\
- \\
-\end{array}$ & & & 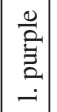 & 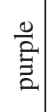 & 总 & 总 \\
\hline$\sum_{\infty}$ & & & & & & & & & & & & & & & & 芯 & 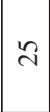 & 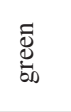 & & & 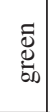 & 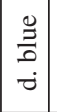 & & $x$ & 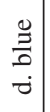 & & & 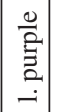 & 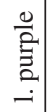 & 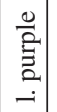 & 产 \\
\hline 紊 & ¿ & 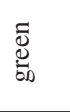 & & $x$ & 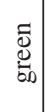 & 总 & & $\times$ & 总 & $x$ & $\star$ & 总 & 总 & 总兽 & 总音 & $\begin{array}{l}\tilde{Z} \\
\tilde{Z} \\
\tilde{L} \\
\end{array}$ & त) & 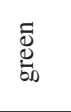 & & $x$ & 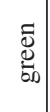 & $\frac{9}{3}$ & $x$ & & $\begin{array}{l}0 \\
ٍ \\
0 \\
-j\end{array}$ & & & 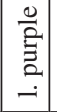 & $\begin{array}{l}\frac{0}{a} \\
\vdots \\
\overline{2}\end{array}$ & 总 & 品 \\
\hline$\sum_{2}^{10}$ & ते & $\begin{array}{l}\bar{\Xi} \\
\stackrel{\Xi}{5} \\
\text { to }\end{array}$ & & $\star$ & 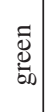 & 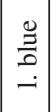 & & & $\begin{array}{l}\stackrel{0}{ٍ ٍ} \\
-\end{array}$ & & $x$ & 号 & $\begin{array}{l}\frac{0}{2} \\
\stackrel{2}{2}\end{array}$ & $\begin{array}{l}\bar{\Xi} \\
\stackrel{0}{0} \\
\stackrel{0}{-}\end{array}$ & 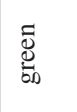 & $\overrightarrow{\tilde{J}}$ & त) & 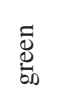 & & & 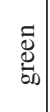 & $\mid$\begin{tabular}{c|}
$\frac{0}{2}$ \\
$\stackrel{2}{2}$ \\
$\stackrel{0}{2}$ \\
$\stackrel{2}{2}$
\end{tabular} & 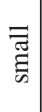 & & 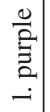 & & & 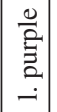 & 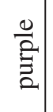 & 产 & 总 \\
\hline 苔 & $\tilde{\sim}$ & 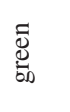 & 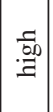 & & 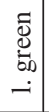 & $\begin{array}{c}\mathscr{g} \\
\stackrel{0}{0} \\
\stackrel{0}{-}\end{array}$ & & & $\stackrel{\substack{\mathscr{m} \\
:}}{=}$ & & & 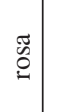 & 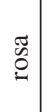 & $\begin{array}{l}\stackrel{0}{\overrightarrow{2}} \\
\stackrel{2}{2}\end{array}$ & 产 & 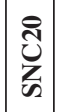 & $\infty$ & 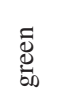 & & 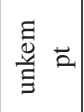 & 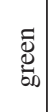 & $\mid$\begin{tabular}{l|}
$\tilde{g}$ \\
0 \\
0
\end{tabular} & & & $\begin{array}{l}\mathscr{o} \\
\stackrel{o}{Q}\end{array}$ & & & 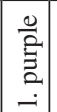 & 总 & $\mid$ & 品 \\
\hline$\sum_{\tilde{s}}^{\tilde{z}}$ & $\vec{\sim}$ & 总 & & & 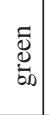 & 足 & $\stackrel{9}{: 00}$ & & $\begin{array}{c}\stackrel{0}{a} \\
\stackrel{0}{a} \\
\stackrel{0}{-}\end{array}$ & $\begin{array}{l}: 2 \\
: 00 \\
0 \\
0\end{array}$ & & 号 & $\begin{array}{l}\frac{0}{2} \\
\vdots \\
\vdots\end{array}$ & 苋 & 总 & $\begin{array}{l}\vec{Z} \\
\bar{Z} \\
\dot{Z}\end{array}$ & $=$ & 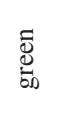 & & & 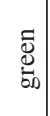 & $\mid \begin{array}{l}\tilde{v} \\
: \\
0\end{array}$ & & & 总 & $\times$ & & 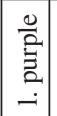 & 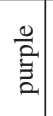 & 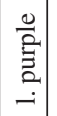 & $\frac{0}{2}$ \\
\hline 苾 & 0 & 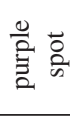 & $\star$ & & $\begin{array}{l}\tilde{\Xi} \\
\bar{\Xi} \\
\vdots \\
0\end{array}$ & 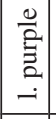 & & & 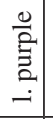 & & & $\frac{0}{\vec{a}}$ & $\begin{array}{l}\stackrel{0}{a} \\
\stackrel{2}{2}\end{array}$ & 产 & 言. & $\begin{array}{l}\infty \\
己 \\
Z \\
\vdots \\
\infty\end{array}$ & $\approx$ & 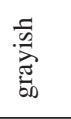 & & & $\begin{array}{l}\bar{D} \\
\bar{D} \\
\dot{\Delta} \\
0\end{array}$ & 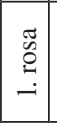 & & & $\begin{array}{c}\stackrel{\mathscr{b}}{0} \\
\stackrel{0}{0} \\
\stackrel{-}{-}\end{array}$ & & & 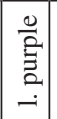 & 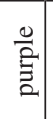 & \begin{tabular}{|c|}
$\frac{0}{2}$ \\
$\stackrel{2}{2}$ \\
$\stackrel{2}{2}$ \\
\end{tabular} & 产 \\
\hline $\bar{z}$ & त) & $\begin{array}{l}\text { 胥 } \\
\text { फू }\end{array}$ & & & $\begin{array}{l}\bar{\Xi} \\
\bar{\Xi} \\
\overline{5}\end{array}$ & $\stackrel{9}{\Xi}$ & $\times$ & & $\stackrel{0}{\frac{0}{0}}$ & $x$ & & $\frac{0}{\frac{0}{2}}$ & 竞 & $\frac{\stackrel{0}{\tilde{z}}}{\mathrm{a}}$ & $\frac{0}{\grave{\Xi}}$ & $\begin{array}{l}0 \\
己 \\
Z \\
Z\end{array}$ & $\because$ & 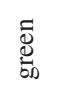 & & $x$ & 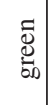 & 咅 & & & $\frac{0}{\frac{7}{3}}$ & $x$ & & 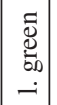 & 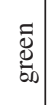 & 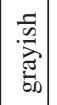 & 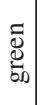 \\
\hline & 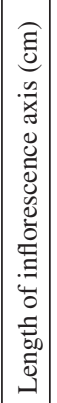 & 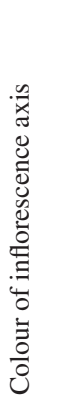 & 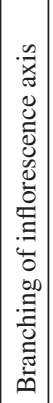 & 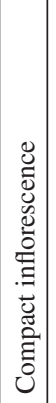 & 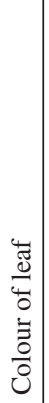 & 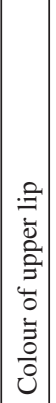 & 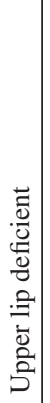 & 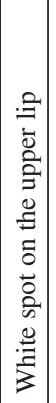 & $\begin{array}{l}: \\
: \\
0 \\
0 \\
0 \\
0 \\
0 \\
\vdots \\
0 \\
0 \\
0\end{array}$ & 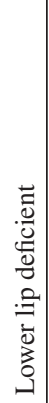 & 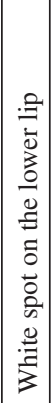 & 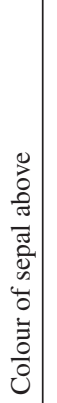 & $\begin{array}{l} \\
0 \\
0 \\
0 \\
0 \\
0 \\
0 \\
0 \\
0 \\
0 \\
\vdots \\
0 \\
0 \\
0\end{array}$ & 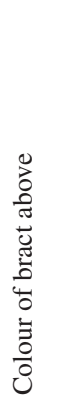 & 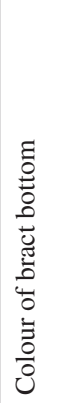 & & 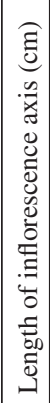 & 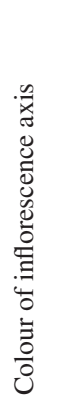 & 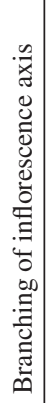 & 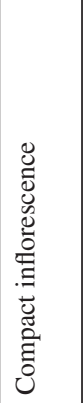 & 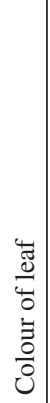 & 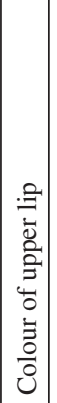 & 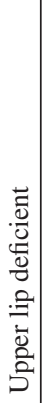 & 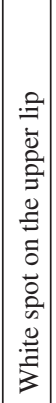 & $\begin{array}{l}: \\
\overline{0} \\
0 \\
0 \\
\overline{0} \\
\vdots \\
\overline{0} \\
0 \\
0\end{array}$ & 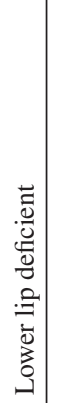 & 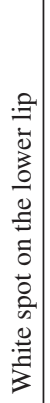 & 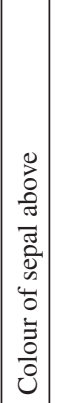 & 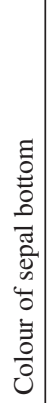 & 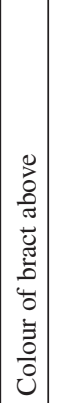 & 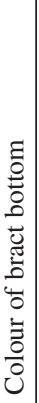 \\
\hline
\end{tabular}


Table 2: Evaluation of the colour versions of Wild sage (DE AGTC DTTI, 2012)

\begin{tabular}{|c|c|c|c|c|c|c|}
\hline Versions & Colour of leaf & $\begin{array}{c}\text { Length of } \\
\text { inflorescence axis }\end{array}$ & $\begin{array}{c}\text { Colour of inflorescence } \\
\text { axis }\end{array}$ & $\begin{array}{c}\text { Form of } \\
\text { inflorescence }\end{array}$ & $\begin{array}{l}\text { Colour of upper } \\
\text { lip }\end{array}$ & Colour of lower lip \\
\hline SN 1 & dark green & middle & green with purple stripe & unkempt & dark purple & dark purple \\
\hline SN 2 & green & middle & green with rosa spot & simple & light purple & light purple \\
\hline SN 3 & light green & long & purple stripes & simple & rosa & rosa \\
\hline SN 4 & green & long & purple stripes & simple & light rosa & rosa \\
\hline SN 5 & light green & middle & green-rosa & simple & blue-purple & purple \\
\hline SN 6 & green & middle & dark rosa & simple & blue-purple & dark blue-purple \\
\hline SN 7 & green & long & green with purple stripe & simple & blue-purple & blue-purple \\
\hline SN 9 & light green & short & $\begin{array}{c}\text { light rosa with green } \\
\text { stripe }\end{array}$ & simple & light blue-purple & light blue-purple \\
\hline SN 11 & light green & middle & green with rosa stripe & simple & light purple & light purple \\
\hline SNC 13 & yellow-green & middle & yellow-green & simple, thin & white & white \\
\hline SNC 14 & green & long & green-purple & simple & light purple & dark purple \\
\hline SNC 15 & light green & middle & yellow-green & simple & white & white \\
\hline SNC16 & light green & middle & green with purple spot & simple & light purple & light purple \\
\hline SNC 17 & green & middle & yellow-green & unkempt & white & white \\
\hline SNC 18 & light green & long & $\begin{array}{c}\text { light green with purple } \\
\text { stripe }^{22}\end{array}$ & simple & purple & dark purple \\
\hline SNC 19 & light green & short & green-purple & simple & light purple & light purple \\
\hline SNC 20 & light green & short & green with rosa spot & unkempt & light purple & dark purple \\
\hline SNC 21 & green & middle & green & simple, solid & purple & purple \\
\hline SNC 22 & green & middle & green with rosa spot & simple & purple & purple \\
\hline SNC 23 & green & long & purple & simple & dark purple & dark purple \\
\hline SNC 24 & dark green & middle & green-purple & simple & dark purple & dark purple \\
\hline SNC 25 & light green & middle & green with purple stripe & simple & dark purple & dark purple \\
\hline SNC 26 & light green & short & green with rosa spot & simple & light purple & light purple \\
\hline SNC 27 & dark green & long & green with purple stripe & simple & dark purple & dark purple \\
\hline SNC 28 & green & long & green with purple spot & simple & dark purple & dark purple \\
\hline SNC 29 & green & short & green with purple stripe & simple & dark purple & dark purple \\
\hline SNC 30 & green & middle & $\begin{array}{c}\text { green with light purple } \\
\text { spot }\end{array}$ & simple & dark purple & light purple \\
\hline SNC 31 & green & long & $\begin{array}{c}\text { green with light purple } \\
\text { spot }\end{array}$ & unkempt & light purple & $\begin{array}{l}\text { light blue with white } \\
\text { stripe }\end{array}$ \\
\hline
\end{tabular}

three basic colours of the upper and lower lip, but in the natural vegetation with random crossing different shades are formed. The length, colour and solidity of the inflorescence axis are also different. Leaves of the white lip versions can be yellowish-green or green.

The yellow-green leaf colour of SNC13 clone is special for matter. It could be combined with other dark green colour of annual and perennial ornamental plants. Because of the light leaf colour and white lip colour of this clone, it can associate with different dark Salvia clones.

The plant's length of inflorescence axis determines the area of its use. The longer inflorescence axis makes Salvia nemorosa decorative as background-plants, as cut flowers. The wild sage with shorter inflorescence axis is preferably suitable as potted plant or bed-border. It is attractive among roses, too.

The SNC28 clone has the longest inflorescence axis, and this is followed by the SNC27 clone. The SN3, SNC14, SNC23 and the SNC31 versions have still inflorescence axis of long length. In literature, it has not been included the colour of inflorescence axis, although this stamp can be also important. The basic colour of the axis is green, sometimes with pink or purple spots, stripes. This is really a special sight. The inflorescence axis of the white lips of versions (SNC13, SNC15, SNC17) has uniformly yellow-tinged colour.

The study of the flower forms shows an interesting result. In literature, the form of lips is not mentioned much more. Some clones have the simple form instead of unkempt lip form (SN1, SNC17, SN20, SNC31), which can be suitable for cut or potted flower.

The location of lip flowers on the inflorescence axis is also an important stamp. The white coloured-lips of SNC13 clone are sparsely placed on the axis, while SNC21 clone has dense lips. Literature does not mention the colour of lower and the upper lip separately. Botanists have not studied this botanical stamp yet. Some clones have shades difference between two lips, which is an important property. The upper lip of the SN4, SN5, SN6, SNC14, SNC18 and SNC20 clones is lighter. The lower lip of the SNC30, SNC31 clones is lighter than the upper one. 


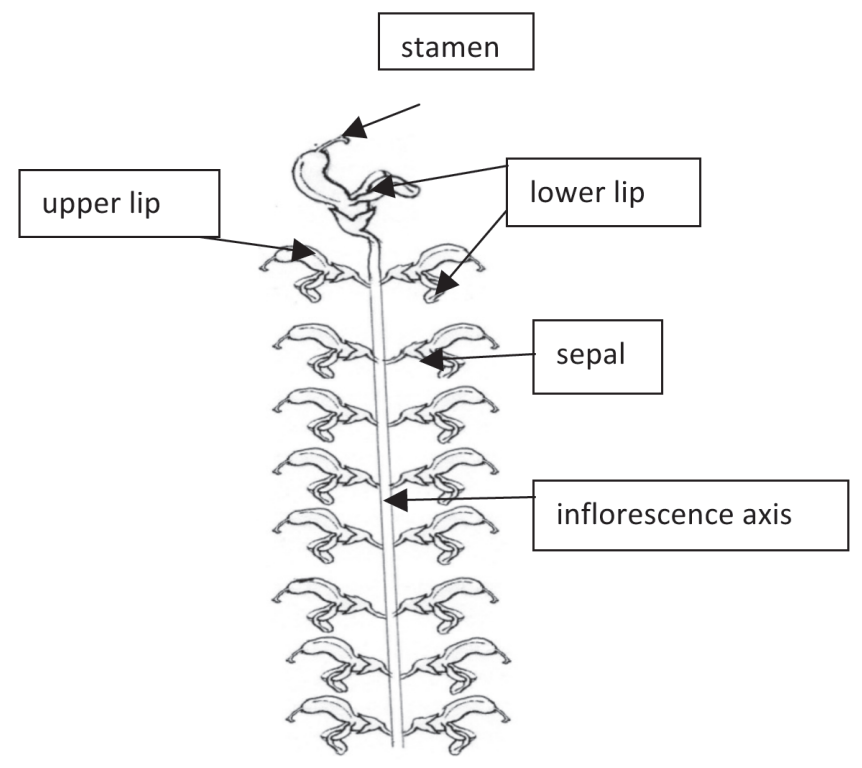

Figure 1: Inflorescence axis of Wild sage (Salvia nemorosa L.) botanical description

Special selection varieties are important on account of the horticultural usability. After planting, we will go on with testing of the different colour and shape variations already collected stems.

\section{Conclusion}

Weather anomalies do more damage also to ornamental planting. Beside ornamental species and varieties of the public planting, it will be necessary to use of the domestic well-changeable climate-tolerant plantspecies. The natural populations of wild sage are still unknown to us colour, their and shape variations are hidden. Beside the three main versions of the clones collected from the natural vegetation, further colour versions have been found. The axial length of inflorescens, colour and brevity of the flowers were also significant differences. The botanists have not tested the sepal and bract from top to bottom yet, which may vary. In 2011, the shoots are planted in field with breeding methods to perform an additional option.

From the collected and morphologically evaluated Salvia variants we want to create a new type of hungaricum series for widespread application. The excellent drought- and frosttolerant Salvia nemorosa belongs to the climate changetolerant perennials. The natural vegetation has been collected, as lusus forms (spontaneously appearing and disapperaring botanical shape and colour versions) from which we have selected new unique shape and colour versions. These properties have not been studied by botanists yet, which give a new direction in using as an ornamental plant. The different shape- and colour versions broaden the variety. We are going to study the valuable versions from botanical-ethnobotanical point of view in the future. The collected, morphologically evaluated Salvia versions create a widespread application for a new type of hungaricum series.

\section{Acknowledgments}

With this written article we want to remember our internationally recognized breeder, Zoltan Kovats, who dealt with this plant enthusiastically in our country by searching and preserving the new versions of these wild plant species.

The present work has been supported by MOP-Biotech Ltd. (Nyíregyháza), and by the Károly Ereky Biotechnology Foundation (Debrecen).

\section{References}

Cervelli, C., Pasini, C., Ruffoni, B., Sacco M., Capponi, A., Liotta, A., Campagna, G., Mascarello, C. (1999): Salvia. In: Cappuro, M.: Specie spontanee in colture florovivaistiche produttive. Centro Servizi Floricoltura Della Regione Liguria. 221-233. p.

Hegi, G. (1926/7): Illustrierte Flora von Mitteleuropa. V. Band, 4. Teil. Dicotyledones. J. F. Lehmanns Verlag, München. 2501-2503 p.

Kaprinyák, T; Koroknai, J.; Zsiláné, A. A.; Szakadát, Gy., Kováts, Z.; Lévai, P. and Fári, M.. (2012): Új ligeti zsálya (Salvia nemorosa L.) színváltozatok kiemelése és jellemzése. In: Agrártudományi Közlemények, 2012/46. 41-44. p

Király, G. (2009): Új magyar füvészkönyv, Magyarország hajtásos növényei, Határozókulcsok. Aggteleki Nemzeti Park Igazgatóság, Jósvafö. 345., 360. p.

Kováts, Z. (2010): Egy expedíció története Debrecentől. A 73 év alatt megváltozott Berettyó- parti elvadult táj felkutatására a ligeti zsálya- Salvia nemorosa L. színváltozatainak begyüjtése céljából Gáborjánig. Kézirat, DE AGTC DTTI, Debrecen. 1-10 p.

Ruffoni, B., Giovanni, A., Amoretti, M., Pricipato, M.C. and Mascarello, C. (2004): In vitro culture of several ornamental and medicinal Salvia species. In: Biotechnology, as Theory and Practice in Horticulture: In vitro culture and Horticultural breeding. 5th IVCHB Symposium, Debrecen. 182 p.

Soó, R. (1968): A magyar flóra és vegetáció rendszertaninövényföldrajzi kézikönyve. III. Akadémiai Kiadó, Budapest. 104$105 \mathrm{p}$. 